\title{
Arme stratégique pour l'entreprise, son histoire
}

\section{Christian Schneider}

\section{OpenEdition}

\section{Journals}

Édition électronique

URL : http://journals.openedition.org/communicationorganisation/1771

DOI : 10.4000/communicationorganisation. 1771

ISSN : 1775-3546

\section{Éditeur}

Presses universitaires de Bordeaux

\section{Édition imprimée}

Date de publication : 1 mai 1995

ISSN : 1168-5549

\section{Référence électronique}

Christian Schneider, "Arme stratégique pour l'entreprise, son histoire », Communication et organisation [En ligne], 7 | 1995, mis en ligne le 26 mars 2012, consulté le 19 avril 2019. URL : http:// journals.openedition.org/communicationorganisation/1771; DOI : 10.4000/ communicationorganisation. 1771

Ce document a été généré automatiquement le 19 avril 2019.

(c) Presses universitaires de Bordeaux 


\title{
Arme stratégique pour l'entreprise, son histoire
}

\author{
Christian Schneider
}

Dans une entreprise à forte culture, l'histoire est omniprésente, même si celle-ci n'est pas constamment visible pour un œil non averti. C'est elle qui confère à la culture cette dimension transversale, en dehors des clivages hiérarchiques, des fonctions et même du temps ; culture librement acceptée, fondée sur le passé mais représentant un ensemble de valeurs permettant d'avoir confiance dans l'avenir.

2 Pour prendre un exemple récent, la culture des Charbonnages de France apparaît indissociable de son histoire et va rythmer l'attitude des mineurs face à la disparition progressive au cours des quinze dernières années, de leurs univers, de leur civilisation, de leur groupe et de sa mémoire collective. Risque permanent de la mine, souvenir des dramatiques coups de grisou, dureté du travail, rôle majeur dans le redémarrage de l'industrie Française au lendemain de la dernière guerre mondiale, sont autant d'éléments communs provenant directement de l'histoire des houillères qui constituent le patrimoine culturel et social des mineurs, du mineur de base à l'ingénieur. Et jusqu'au bout cette culture fondée sur l'histoire aura été le ciment des actions incessantes en vue de défendre, certes l'emploi des régions minières, mais aussi l'identité des hommes.

3 À côté des aspects internes, l'histoire marque de son empreinte la perception qu'ont de l'entreprise et de ses productions, clients, médias, leaders d'opinion, et, de façon plus générale tous ses partenaires privés et publics. Élément de positionnement, caution, facteur de valorisation et de réputation, l'histoire participe de façon majeure à l'image de l'entreprise et par suite à sa vie.

4 Moteur puissant en interne et externe, l'histoire de l'entreprise peut aussi connaître, des dérives d'autant plus inquiétantes qu'elles ne sont pas visibles et ont tous les aspects de la vérité historique. Les États comme les entreprises peuvent en effet être tentés de s'approprier ce formidable outil et de le façonner de façon à accroître son efficacité. La gamme est ainsi large des moyens, qui de la simple mise en valeur des faits positifs à la construction ex nihilo à des fins de désinformation d'une "fausse histoire ", séparent «l'histoire historique » (celle qui relève d'une démarche impartiale de type universitaire) 
de l'histoire voulue par l'entreprise. L'image est un capital qui se gère; à cet égard l'histoire est un élément majeur et apparaît donc comme un véritable outil de management, voire comme une arme spécifique qu'il convient de perfectionner et d'utiliser. La distance est donc grande, qui sépare la conception du chercheur universitaire de celle du directeur de la communication, chargé de mettre en œuvre une histoire « voulue » mais néanmoins crédible et justifiable...

Outil de management, l'histoire d'entreprise répond à quantité de besoins fondamentaux, tant individuels que collectifs, ce qui lui donne donc une légitimité certaine, même si cette histoire est en partie réécrite dans le but de mieux servir les intérêts de l'entreprise, l'écart avec la réalité dépendant d'une part de l'éthique des dirigeants et d'autre part de la crédibilité à conserver vis-à-vis des publics visés dont les salariés.

En ce qui concerne l'écriture de l'histoire de l'entreprise, nous distinguerons deux approches.

7 La première que nous appellerons «scientifique" se caractérise par la volonté de recueillir le maximum d'informations, tout ce qui peut illustrer l'évolution de l'entreprise et expliquer ses réussites comme ses échecs. La démarche, assez rare il est vrai, est authentique, transparente et ouverte : il s'agit d'assumer son histoire mais aussi d'en tirer des enseignements pour l'avenir.

La deuxième peut être qualifiée " d'utilitaire »; l'objectif réel est de se servir de l'histoire comme d'un outil de management; l'outil est alors spécialement forgé, ce qui a pour conséquence la modification, voire l'élimination de certains aspects de l'histoire et de son contexte. C'est à travers cette deuxième démarche, beaucoup plus répandue, que nous proposons d'aborder le sujet. Pour ce faire, nous avons choisi non pas de tenter une analyse systématique de tous les facteurs intervenant mais de procéder par une d'éclairages permettant de photographier sous des angles privilégiés le phénomène global.

\section{Historiens et hagiographes}

9 Pour une entreprise souhaitant se servir de son histoire, la première étape consiste précisément à établir cette histoire, histoire «vraie» des faits, la plus exhaustive possible, exempte d'interprétations. Cette méthode ne se distingue nullement de celle appliquée par l'historien professionnel, auquel il peut d'ailleurs être fait appel. On peut observer que dans la majorité des cas la phase de recherche est simplifiée par l'accès facile aux archives de l'entreprise et le fait de pouvoir encore rencontrer des témoins (retraités par exemple) qui à leur tour pourront indiquer d'autres sources (livres oubliés, événements ayant été relaté dans la presse de l'époque...). Cette première phase est généralement confiée à un collaborateur extérieur qui toutefois est rétribué pour une recherche et non pas pour la rédaction de l'histoire de l'entreprise. La distinction est d'importance car le collaborateur historien n'aura aucun droit de regard sur l'usage qui sera fait ensuite de son travail; son nom ne sera pas cité; du fait d'un tel contrat, l'entreprise est propriétaire de la recherche effectuée et garde une liberté totale.

10 À partir de ce matériau brut, la direction générale peut déjà avoir une première opinion sur la richesse (ou au contraire le caractère affreusement plat...) de son histoire, les points valorisants, les échecs, voire les faits honteux. Il peut également surgir du passé des expériences vécues et oubliées, qui présentent un grand intérêt de différents points 
de vue (technologique, conception du travail en équipe, pertinence de la réaction face à une situation de crise...). Même si l'histoire de l'entreprise n'est jamais écrite, la recherche réalisée aura permis à la direction générale, d'être éclairée et de ne pas êre surprise si un jour un trait du passé resurgit dans la presse ou au sein même de l'entreprise. Une telle recherche qui n'engage nullement l'avenir est donc d'autant plus justifiée qu'elle est d'un coût généralement abordable.

11 Au stade suivant, à partir de ce matériau brut, va réellement s'édifier l'histoire de l'entreprise selon un processus complexe dans lequel la direction générale joue un rôle majeur, même si apparemment c'est la direction de la communication qui est impliquée ; quant à l'historien, il est généralement absent.

12 Une variante concerne l'historien que l'entreprise rémunère pour qu'il publie sous son nom et chez un éditeur connu (si possible) une histoire de l'entreprise que celle ci aura préalablement contrôlée. C'est en quelque sorte une adaptation de la technique du "nègre ", pratiquée par d'aucuns. Nous nous rapprochons ici du domaine si célèbre au temps de la guerre froide de la propagande souterraine menée par des agents d'influence.

Le problème se pose de façon toute différente pour le véritable historien, indépendant, qui souhaite publier sous son nom et en toute liberté une histoire d'entreprise. La démarche s'apparente à celle du journaliste qui mène une enquête : il est en général reçu, mais on ne lui dit pas tout et on ne lui montre pas tout. Par exemple l'accès aux archives n'est que partiel, l'entreprise n'ayant aucun intérêt à transmettre des documents susceptibles de servir de base à des développements négatifs qu'elle ne pourra en aucun cas maîtriser. Cas limite, l'œuvre de l'historien remet en cause la saga officielle de l'entreprise telle que diffusée par celle-ci depuis des années... Intéressant problème de crise à gérer par la direction de la communication !

Mais dans la grande majorité des cas, c'est l'entreprise qui commandite les recherches historiques la concernant et c'est elle qui, in fine, orientera la vérité.

L'histoire et l'hagiographie n'ont pas grand chose en commun si ce n'est les faits bruts, avant qu'ils n'aient donné lieu à rédaction ou à transformation. La première discipline se veut indépendante de tout organisation et de toute entreprise; la deuxième est dans le cas qui nous intéresse la traduction intelligente des faits dans le but de servir directement la stratégie de l'entreprise. Et c'est précisément l'intelligence de la traduction qui fait que l'hagiographie est invisible et prend l'apparence de l'histoire. Entre les deux extrêmes, il existe quantité de formes intermédiaires, l'entreprise ayant néanmoins plus tendance à choisir un hagiographe, intégré ou non, plutôt qu'un historien pour écrire une histoire qui lui soit utile.

16 Au-delà de l'hagiographie commence un domaine inquiétant, celui de la propagande et de l'histoire totalement, artificiellement créée. Les exemples abondent dans le nazisme et les régimes totalitaires et concernent aussi bien l'histoire « officielle» mise au point par ces régimes que les entreprises existant dans lesdits régimes. Il n'est pas sans intérêt de se pencher sur un exemple qui relève précisément de la manipulation historique et qui concerne l'annexion et la transformation par le tristement célèbre binôme Hitler Goebbels de l'ancienne mythologie des Germains et des Scandinaves que l'on connait surtout à travers les traces laissées en Scandinavie et les écrits trouvés en Islande.

À partir de signes symboliques comme les runes, à la fois lettres et symboles pratiquement inconnus sauf de quelques spécialistes, le régime nazi a réussi à façonner une véritable histoire dont les traits dominants étaient le courage indomptable des 
guerriers, et la supériorité ethnique de la race. La mise en scène que ne renieraient pas des professionnels d'aujourd'hui, àbase de gigantesques manifestations de nuit animées par une illumination appropriée et accompagnées par une musique évocatrice participait àl'édification du mythe. La croix gammée et le logo des SS, tous deux issus d'une stylisation de runes s'imposait et symbolisait le nouveau régime et les valeurs historiques qu'il s'était crée. En quelques années l'ancrage germanique était réalisé et un lien fort entre anciens Germains et Allemands du 3ème Reich établi. La démarche est bien celle de la propagande assise sur une histoire revue et corrigée, liée àdes symboles visuels forts.

\section{Depuis la plus haute antiquité}

tout temps l'histoire d'un groupe a servi de ciment à la culture, à l'identité, à la cohésion,àla civilisation de ce groupe, que celui-ci soit une tribune citée, une nation ou une autre entité. A cet égard, il n'y a pas de différence entre un groupe et une entreprise dans son sens économique actuel. Simplement cette dernière ne concerne ses collaborateurs que pour le temps qu'ils y travaillent et non pour la totalité des heures.

Les valeurs en commun, les exploits des membres du clan, vivants mais aussi ancêtres, en bref, l'histoire participent directement à cette culture commune. Mieux que des lois écrites le respect de la tradition librement acceptée pérennise le système; l'histoire devient mythe et tant que les valeurs du groupe ne sont pas récusées, il n'y a aucune remise en cause des comportements. Qui plus est, au fur

et à mesure que les faits historiques de référence deviennent plus anciens, la légende plus ou moins télécommandée, se substitue à eux, les embellit et les transforme en symboles du clan.

21 Après les premiers combats contre les Spartiates dans la guerre du Péloponnèse, Périclès fut désigné (se fit désigner...) pour faire l'éloge des premiers soldats d'Athènes morts pour la patrie et profita de l'occasion pour dynamiser armée et population dans un discours resté célèbre, qui se réfère aux valeurs communes et à l'histoire de la cité. Thucydide rapporte ce discours ${ }^{1}$

"...Je commencerai donc par nos aïeux. Car il est juste et équitable, dans de telles circonstances de leur faire l'hommage d'un souvenir. Cette contrée que sans interruption ont habité des gens de même race, est passée de mains en mains jusqu'à ce jour, en sauvegardant grâce à leur valeur sa liberté. Ils méritent des éloges mais nos pères en méritent davantage encore. A l'héritage qu'ils avaient reçu ils ont ajouté et nous ont légué, au prix de mille labeurs la puissance que nous possédons. Nous l'avons accrue, nous qui vivons encore et qui sommes parvenus à la pleine maturité...

Je me suis étendu sur les mérites de notre cité, car je voulais vous montrer que la partie n'est pas égale entre nous et ceux qui ne jouissent d'aucun de ces avantages et étayer de preuves l'éloge des hommes qui font l'objet de ce discours. La gloire de la république, qui m'a inspiré, éclate dans la valeur de ces soldats et de leurs pareils. Leurs actes sont à la hauteur de leur réputation...».

Plus près de nous, au milieu du $\mathrm{xx}^{\mathrm{e}}$ siècle, on constate qu'avant même la qualité de leur entrainement, ce qui fait la force des unités d'élite d'une armée, ce sont les références aux exploits passés, à la "différence ", un esprit de corps très fort un attachement profond aux traditions, aux modes et au langage propres de l'unité. En un mot, c'est la culture de ladite unité, très imprégnée de son histoire qui est déterminante. Mais cette histoire est sélective car ce qui est présent dans les esprits, ce sont les exploits et non pas les jours 
moins glorieux et ceci pour une raison évidente: les chefs successifs de ces unités cultivent ces épisodes qui servent d'exemple ; A contrario les autres éléments de l'histoire même s'ils ne sont pas occultés, finissent par disparaître d'eux-mêmes.

Pendant la dernière guerre mondiale, certaines entreprises chimiques Allemandes ont participé activement à la mise au point des gaz utilisés dans le génocide des camps de concentration. On constate aujourd'hui que cette « référence technique » et cette période de l'histoire ont été totalement occultées par les entreprises actuelles concernées. Un tel oubli permet d'écrire une autre histoire, nettement plus favorable à la création d'une image d'entreprise directement exploitable.

L'amnésie voulue dans l'histoire de l'entreprise permet donc, souvent avec le consensus de ceux qui savent («ne remuons pas de tristes souvenirs»), d'évacuer certaines périodes. Qui se souvient en France, hormis les personnes âgées et les historiens, des raisons qui ont amené à tort ou à raison (notre propos n'étant nullement de prendre parti), la nationalisation de Renault ? L'histoire que Renault retient ne met évidemment pas l'accent sur ces périodes troubles, et s'appuie avec raison aussi bien sur l'épopée d'avant-guerre que sur les succès de tous ordres enregistrés depuis des années. On constate ici encore que l'histoire de l'entreprise a été bâtie à partir des points forts et que, finalement, pour l'opinion publique (et sans doute pour le personnel), les épisodes moins glorieux n'ont jamais existé. Ces quelques exemples mettent en valeur trois points :

28 - l'importance de l'histoire pour souder un groupe et lui faire partager une culture commune

29 - la tendance presque systématique pour les dirigeants à s'en servir comme d'un levier augmentant la cohéson et la puissance du groupe, et par voie de conséquence leur propre pouvoir

30 - la technique généralement retenue, consistant à ne retenir dans la véritable histoire que les « morceaux choisis » voire à délibérément occulter certains faits.

\section{Répondre à des besoins d'identité}

31 De façon très naturelle, les hommes d'un groupe ont besoin de croire à des valeurs supérieures et communes. Ces valeurs rassurent, fortifient l'individu et dans une certaine mesure, donnent un sens à leur vie, pour ceux qui n'ont pas une personnalité marquée. Plus la situation est difficile, plus le besoin de croire à des valeurs communes s'exprime. L'histoire est ainsi une mine inépuisable dans laquelle l'individu et le groupe finissent collectivement par trouver de nouvelles raisons d'espérer ainsi que la force de surmonter la situation.

Le comportement des salariés d'une entreprise ne diffère pas sensiblement de celui des membres d'une tribu ou des citoyens d'un pays. Il convient toutefois de remarquer que le raisonnement ne s'applique qu'au cas des entreprises à forte personnalité dont les collaborateurs sont impliqués et s'identifient fortement à leur société. Les autres entreprises, celles qui n'ont pas de forte culture (or culture et histoire sont intimement liées) ne déclenchent généralement pas de telles réactions parmi leurs collaborateurs. Ces entreprises là sont à la limite des lieux neutres dénués d'affectivité dans lesquels les collaborateurs échangent leur travail contre une rémunération. 
$33 \mathrm{Au}$ delà des réactions collectives des salariés par rapport à leur entreprise, à sa culture et à son histoire, il existe de multiples raisons qui expliquent les attitudes individuelles de chacun : depuis le souci de valorisation de soi-même via l'entreprise jusqu'à la création d'un lien mythique entre l'homme et son entreprise, lien qui fait penser au mythe du père ou à l'environnement dans l'industrie japonaise d'il y a une vingtaine d'années. Ce type de comportement tend tout naturellement à faire que l'histoire de l'entreprise est embellie, un peu à la façon des chansons de geste, et cela sans même d'intervention volontariste de la direction générale pour « arranger » l'histoire de façon à la rendre plus implicante et plus fédérative vis-à-vis des collaborateurs.

34 A titre d'illustration de ce lien très fort entre le collaborateur et l'entreprise, lien fondé en grande partie sur l'histoire, nous, citerons l'épisode suivant, véridique, intervenu il y a une dizaine d'années: un cadre supérieur du groupe Fait licencié suite à une restructuration a aussitôt entrepris des recherches historiques sur cette firme, s'est intéressé aux vieilles voitures de la marque, a collaboré à des journaux et des revues spécialisées en tant qu'expert... Pour lui, ce n'est pas Fiat qui l'avait licencié, c'était « $\mathrm{x}$ " (directeur du personnel). La rupture du contrat de travail n'avait en rien changé le lien qui existait entre ce cadre, Fiat, et l'histoire de ce groupe.

Dans d'autres secteurs, à la SNCF notamment, on a pu observer des comportements relevant du même modèle.

36 A la limite, pour la direction générale, un tel attachement à l'histoire de l'entreprise et à ses valeurs peut constituer un frein important au développement du fait du refus des hommes de rompre brutalement avec le passé. Ceci est particulièrement vrai dans le cas de fusions ou de rachats d'entreprises.

Dans un récent article paru dans Le Monde, Pierre Le Hir se livre à une étude diagnostique fort intéressante concernant Merlin Gérin et ses observations relatives au comportement des salariés vis-à-vis de leur entreprise qui a perdu son identité en changeant de nom et de propriétaire, éclairent tout particulièrement le débat ${ }^{2}$ :

"Bienvenue aux anciens de Schneider Electric! a lancé l'animateur, qui n'y entendait pas malice, en ouvrant la fête de fin d'année des retraités. Le présentateur mal inspiré, conspué par le millier de personnes présentes, n'a pu poursuivre plus avant. Ces anciens là n'étaient pas issus de Schneider Electric mais de Merlin Gerin...

Trois ans après l'absorption du premier employeur isérois (Merlin Gérin) par le groupe Schneider, un an après la fusion avec Telemécanique au sein de la nouvelle société Schneider Electric, son personnel reste partagé selon une ligne de fracture linguistique - ce sont souvent les plus résistantes- que n'avaient sans doute pas prévu les partisans de ces restructurations. Les cadres et les nouveaux embauchés disent Schneider Electric, tous les autres continuent de dire Merlin Gérin, assure un employé. Pour beaucoup, il ne s'agit pas d'un simple changement de nom mais d'une véritable perte d'identité...

...Se défendant de céder au passéisme, mais désireux de ne pas perdre toute trace d'un passé qu'ils croient "constitutif de leur avenir", les salariés se sont engagés depuis deux ans, avec la commission culturelle de leur comité d'entreprise dans un travail de mémoire collective exemplaire, dont ils n'avaient pas imaginé qu'il prendrait tant d'ampleur, ni qu'il rencontrerait un si vifécho. "

41 Il apparait donc clairement que l'une des fonctions premières de l'histoire de l'entreprise est de nourrir une culture collective librement acceptée par chacun des collaborateurs, l'acceptation se transformant le plus souvent en appropriation. 
Face à ce constat qui est déjà ancien, la tentation est grande pour la direction générale d'utiliser au mieux cet exceptionnel levier de management. Et quoi de plus naturel que d'agir dans le sens du mouvement, c'est-à-dire d'essayer de gommer les aspects négatifs et de valoriser les aspects positifs, voire d'en faire des emblêmes.

Une telle démarche qui n'est pas rare dans les grandes entreprises disposant d'une direction de la communication musclée permet alors à la direction générale d'en tirer un profit maximum :

\section{- valorisation de l'image externe}

- forte implication des salariés de l'entreprise

- existence d'une référence commune contre laquelle il est tacitement exclu que salariés, syndicats et comités d'entreprise s'insurgent. En d'autres termes, c'est une force de management.

\section{Optimiser l'histoire}

47 Depuis des années les entreprises s'efforcent de mettre au point des solutions opérationnelles permettant en interne de développer une culture commune et un esprit d'entreprise, de faire croître le sentiment de fierté d'appartenir à l'entreprise, de faire en sorte que les collaborateurs soient plus impliqués et deviennent des forces de proposition.

Projet d'entreprise, tentatives de management à la japonaise (référence au modèle antérieur) développement de cercles de qualité, paternalisme "éclairé » sont autant d'approches du même problème. Une constante émerge toutefois: dans tous, les cas l'histoire de l'entreprise est largement valorisée et se trouve être à la base de la culture commune à laquelle il est fait référence.

9 Au-delà de l'histoire et de la culture de l'entreprise, on a vu apparaître progressivement au cours des deux dernières décennies le concept d'image d'entreprise ${ }^{3}$, cette image intégrant tout à la fois images de marques commerciales, résultats économiques et financiers, faculté d'innovation, diversité des marchés, style de management, social et quantité d'autres composantes. Or cette image globale, considérée comme une véritable immobilisation occulte, susceptible d'augmenter dans une large proportion la valeur réelle de l'actif est étroitement liée à l'histoire de l'entreprise et s'appuie en partie sur elle.

Un cas particulier que l'on rencontre souvent dans les entreprises Américaines est celui de la «success story». En parfaite harmonie avec la civilisation et le mode de pensée Américains, l'entreprise raconte la succession d'étapes heureuses (il n'y a jamais d'échecs...) qui ont jalonné son développement depuis sa fondation. Généralement le mythe du fondateur, pratiquement déifié, et de ses successeurs est pleinement associé à l'épopée. A titre d'illustration et pour ne prendre que des exemples très récents, on peut citer l'histoire de Microsoft liée à celle de son président, Bill Gates, ou encore celle de Gateway, leader Américain sur le marché des ventes par correspondance de PC, qui artificiellement se réfere à des valeurs rurales mais aussi à la technologie de pointe : on souligne en effet dans l'histoire qui est officiellement diffusée que le fondateur dont la famille possédait une ferme d'élevage de bétail (référence aux racines profondes des Américains)est resté dans cette ferme pour créer son entreprise, qui en moins de dix ans 
a réussi à prendre une position de leader du fait de l'exceptionnelle qualité de ses produits et de son service (référence au mythe du succès).

51 Ces "success stories", soigneusement écrites, finissent par s'imposer à tous et au personnel notamment ; ceux qui restent dans l'entreprise sont par définition d'accord et y croient d'autant plus que la communication externe faite par la société (en particulier par voie publicitaire) complète le dispositif.

Dans notre vieux continent, pour des entreprises anciennes mais ayant une gestion moderne, l'histoire de la Société est devenu un élément d'importance sur lequel s'appuie la direction générale. Quantité de fonctions sont parties prenantes :

53 Le management et la DRH se servent du passé comme d'un levier pour développer culture d'entreprise, sentiment d'appartenance, fierté, volonté commune d'être les meilleurs (être à la hauteur de ce qu'ont fait les prédécesseurs, ce qui rappelle d'ailleurs le discours de Périclès aux Athéniens)... Ces valeurs sous tendent des valeurs industrielles plus prosaïques telles que productivité, qualité, organisation, efficacité, responsabilité... Un excellent exemple de cette démarche nous est fourni par le secteur du BTP. Quand Francis Bouygues, était encore à la tête de son groupe, la culture y était extrêmement forte s'appuyant à la fois sur l'histoire (les prouesses sur les chantiers) et les hommes (ceux qui avaient "fait» ces chantiers). Les anciens, "Compagnons du Minorange", n'avaient nul besoin d'être cadres pour pouvoir s'adresser en direct au président; une sorte de hiérarchie parallèle fondée sur le passé entretenait ainsi le mythe Bouygues pour être encore meilleur dans le futur. Il va de soi que dans cette saga autotransmise, seuls les points positifs avaient droit de cité. Dans ce type de situation, l'utilisation de l'histoire de l'entreprise pour conforter sa culture engendre une personnalité collective qui finit par se substituer aux personnalités individuelles; tout devient un défi collectif qu'il faut relever pour être digne de son histoire.

La direction du marketing ne manque pas de se servir de certains points de l'histoire à titre de caution, de référence et aussi pour se distinguer des concurrents. Toute l'intelligence de la démarche consiste à utiliser le passé dans un contexte de dynamisme tourné vers l'avenir. Et à titre de bénéfice marginal, le discours tenu vis à vis de l'extérieur vient renforcer la culture du personnel.

La direction de la production profite de la culture des collaborateurs qui rend plus facile la mise en place de structures visant à améliorer les conditions de production, par exemple l'introduction de cercles de qualité.

Il faut toutefois se garder de croire que l'histoire de l'entreprise suffit à entrainer les conséquences bénéfiques que nous venons d'évoquer. Quatre conditions nous semblent devoir être respectées :

L'histoire doit être écrite de telle façon que seuls les aspects positifs apparaissent, mais que les aspects "oubliés» ne soient pas susceptibles de revenir en boomerang en déclenchant une polémique, en devenant une «affaire», ou plus simplement en faisant prendre conscience au personnel et aux partenaires extérieurs que l'entreprise a travesti la vérité. En dehors de considérations éthiques, il faut donc savoir anticiper et décider en fonction du risque analysé.

58 L'histoire doit faire l'objet d'une véritable appropriation par le personnel et être crue par les publics externes; ceci requiert un environnement favorable et l'utilisation de catalyseurs sur mesure. 
59 L'histoire doit être totalement intégrée dans la stratégie de communication, interne et externe, de façon à provoquer des réactions de synergie avev les actions prévues par ailleurs.

60 L'histoire ne doit pas être vécue par le personnel ou perçue à l'extérieur sous un angle passéiste (risque de l'effet «musée ») mais comme un patrimoine mettant en valeur les facultés d'adaptation et d'innovation de l'entreprise.

61 En bref l'histoire est pour une direction générale une arme stratégique et, comme pour toute arme de cette nature, il convient de la définir et de la construire sur mesure. C'est donc très logiquement en fonction d'un cahier des charges clair et en fonction d'une volonté stratégique précise que la direction de la communication va faire écrire sur le papier l'histoire de l'entreprise, tâche qui relève généralement d'un professionnel de la communication et non d'un historien. On est ainsi très loin de la véritable démarche d'historien, mais pour autant il ne faut pas crier abusivement à la mystification ou à la manipulation. Certes les épisodes peu glorieux sont gommés (mais, quelle est l'entreprise qui ne ment pas par omission dans ses relations commerciales ?) et les étapes glorieuses mises en valeur; toutefois, dans la plupart des cas, il n'y a pas trahison mais simple adaptation des faits historiques aux objectifs de l'entreprise. Pour la direction générale, l'histoire de l'entreprise est un arme puissante qu'il faut maîtriser et non pas un témoignage historique à verser dans le patrimoine culturel régional ou national.

\section{De l'histoire de l'entreprise à la propagande, la dérive}

62 Comme nous l'avons déjà souligné, la véritable histoire de l'entreprise, celle qu'aurait écrite un historien ne correspond qu'exceptionnellement à celle qui est utilisée par ladite entreprise. Toute la question est donc de savoir quelle est la limite au delà de laquelle une adaptation devient une manipulation dans laquelle la vérité historique est minoritaire, voire disparait totalement. Passée cette limite qui est principalement éthique, on entre dans le domaine de la désinformation et de la propagande. L'irruption dans ce dernier univers est d'autant plus pernicieuse qu'elle est difficilement repérable par le commun des mortels : l'histoire "présentée» a tous les aspects de l'authenticité alors même qu'elle en est aux antipodes.

63 Le phénomène est ancien. Jean-Paul Gourevitch ${ }^{4}$ rapporte le rôle joué par Mécène vis à vis de Virgile pour amener ce dernier à mettre son talent au service d'Auguste :

64 "Virgile avait été finalement convaincu de tenter de réconcilier les deux moitiés de l'empire romain, les provinces occidentales et les empires hellénistiques en leur trouvant une origine commune qui les justifiait. Ce fut l'Enéide qui rattachait Rome à la filiation Troyenne et établissait Auguste comme le descendant d'Enée et le garant de la continuité de la dynastie. On conçoit que l'empereur, quoique débordé par sa tâche, prit le temps d'écrire personnellement à Virgile pour lui demander d'achever au plus vite son épopée. La fonction du mythe est bien de préserver un pouvoir et de faire écran... Il (le mythe) rend ainsi l'histoire exemplaire, l'arrache au temps et à l'usure pour l'instituer dans l'ordre de l'éternité et de la permanence, comme le montrera de façon encore plus éclatante le culte de l'empereur. "

Hitler ne procédait pas autrement quand il établissait par " savants » et « universitaires » interposés une filiation directe entre les anciens Germains et Scandinaves et le III $^{\mathrm{e}}$ Reich, filiation destinée à démontrer la validité du régime et de ses idées, l'ancienneté et 
l'authenticité(!) de ses racines, la réalité de la supériorité de la race, opposée aux autres (Untermenschen).

Nous sommes certes ici dans le domaine historique qui peut sembler très loin de celui des entreprises. Mais, dans des périodes très récentes, la désinformation, à base d'histoire et de soi disant faits n'a-t-elle pas concerné tout une série d'entreprises d'au delà de l'exrideau de fer, y compris celles traitant du nucléaire. Les enseignements de l'histoire, en matière de désinformation peuvent éclairer la limite que l'entreprise ne doit pas franchir quand elle rédige sa propre histoire et tente d'en tirer profit.

\section{En conclusion, une histoire engagée}

Dans la littérature, on parle d'auteurs engagés. De la même façon on pourrait parler à propos d'histoire d'entreprise d'une histoire engagée. L'entreprise n'est pas un laboratoire universitaire au sein duquel des thésards vont mener une recherche historique et publier, avec le mécénat de l'entreprise le fruit de leurs travaux

L'entreprise n'est d'autre part nullement tenue d'écrire son histoire. Si elle le fait ou si, elle réécrit la recherche qu'elle a demandé à un historien de métier, c'est que cette démarche peut lui servir et lui fournir une arme dans la compétition économique. L'histoire de l'entreprise doit ainsi contribuer comme outil de mangement ou de référence à la conquête des marchés et au profit. Il ne faudrait pas pour autant oublier le rôle social entrainé par la démarche; celle-ci, même engagée, ne peut pas ne pas engendrer dans le groupe concerné un esprit «maison » s'appuyant sur l'histoire, esprit qui fait, en dépit des critiques, que l'environnement des salariés est beaucoup plus humain qu'en l'absence de toute culture. Une histoire d'entreprise « engagée ", donc non impartiale vaut finalement mieux que pas d'histoire du tout.

69 En conclusion, on ne peut validement comparer histoire et histoire d'entreprise. La première est désinteressée, authentique et ne poursuit qu'un but, améliorer les connaissances et la culture de l'homme ; la seconde, engagée vise en tout premier lieu un meilleur rendement de l'entreprise, même si par rebond elle peut avoir des effets bénéfiques pour les salariés ou si marginalement elle peut réveler des faits inconnus sur le plan de la véritable histoire et de la sorte l'enrichir.

\section{NOTES}

1. Thucydide, histoire de la guerre du Péloponnèse

2. "La mémoire disjonctée de Merlin Gerin », Pierre Le Hir. Le Monde du 22 février 1995

3. Communication, nouvelle fonction stratégique de l'entreprise, Christian Schneider, Delmas 1993, $2^{\mathrm{e}}$ édition

4. La propagande dans tous ses états, Jean-Paul Gourevitch, Flammarion 1981. 


\section{RÉSUMÉS}

L'histoire et l'histoire d'entreprise sont deux genres totalement différents ; la première relève de la culture et s'efforce d'être "vraie » alors que la seconde est un outil au service de la direction générale, outil qui agit à travers la culture d'entreprise. Valoriser l'image de l'entreprise à l'extérieur, améliorer le climat interne et l'implication des salariés sont les véritables enjeux de la démarche. L'histoire de l'entreprise est donc façonnée dans ce but : les épisodes glorieux sont mis en valeur alors que les échecs ou les périodes moins claires sont gommés. Mais la « réécriture » de l'histoire de l'entreprise présente des risques potentiels. Au-delà d'une certaine limite, essentiellement éthique, commence le domaine de la propagande et de la désinformation. Néanmoins, en dépit des «arrangements" apportés, l'histoire de l'entreprise entraîne généralement des conséquences positives pour l'entreprise elle-même et ses salariés.

History and business history are two completly differents things. The first belongs to the field of culture and tries to be "true" while the second is an instrument $\mathrm{n}$ the hand general management who wield it through the enterprise culture. What is at stake in this stategy is the idea of burnishing the image of the entreprise on the outside and of improving both the atmosphere inside the company and the role that the workers play. The history of a business is therefore shaped with this goal in mind: glorious episodes are highlighted whereas failures or more sombre periods are glossed over. But « rewriting » business history presents a few potential risks. Beyond a certain limit, which is basically ethical, lie propaganda and disinformation. Nevertheless, despite certain «arrangements » the history of a business generally bring about positive consequences for the business itself and for its workers.

\section{AUTEUR}

\section{CHRISTIAN SCHNEIDER}

Ingénieur de formation, diplômé de l'Institut d'Administration des Entreprises de Paris, l'auteur, après avoir été consultant en agence-conseil et directeur de la communication de grandes entreprises est aujourd'hui ingénieur - conseil et consultant en communication indépendant. Il s'est spécialisé dans le domaine de la communication interne, du management et l'obtention d'effets de synergie avec la communication externe. Christian Schneider est aussi enseignant à l'IAE de Paris (Université de Paris 1 Panthéon Sorbonne. Il a publié en 1993 chez Delmas « Communication, nouvelle fonction stratégique de l'entreprise » (2e édition).). 